\title{
A green-analytical chemistry method for agrochemical- residue analysis in vegetables
}

Maira D. Carabajal, Juan A. Arancibia,** Graciela M. Escandar*

Instituto de Química Rosario (CONICET-UNR), Facultad de Ciencias

Bioquímicas y Farmacéuticas, Universidad Nacional de Rosario, Suipacha

531 (2000) Rosario, Argentina. E-mail: escandar@iquir-conicet.gov.ar 


\begin{abstract}
Ten agrochemicals, including fungicides, insecticides, herbicides and a plant growth regulator, were quantified at part per billion levels in complex matrices using a greenanalytical chemistry (GAC) method. Liquid chromatography with dual UV/diode array (DAD) and fluorescence (FLD) detections was carried out in a single run, and the secondorder DAD-elution time and FLD-elution time data obtained were treated with MCR-ALS (multivariate curve resolution/alternating least-squares) algorithm. In this way, while analytes are measured through their more appropriate (absorbance and/or fluorescence) signals, chemometric treatment of the corresponding matrices allows the resolution of total or partial overlapped bands, and to overcome the presence of interferences in real samples. In this work, FLD-elution time second-order data were obtained for the first time at two excitation wavelengths, improving the sensitivity of fluorescent analytes. The approach was successfully applied to in land cultivated vegetables, including mushroom, lettuce, alfalfa sprout, cucumber, and celery.
\end{abstract}

Keywords: UV-fluorescence-liquid chromatography; Second-order calibration; Agrochemicals; Vegetables samples. 


\section{Introduction}

The unquestionable benefits of the use of pesticides and growth activators in the agro-economy are contrasted with the disadvantages of the widespread human exposure to their residues in fruits and vegetables. Environmental agencies around the world have established legal directives to control their concentrations by defining suitable maximum residue levels (MRL), which refer to the highest level of a pesticide residue that is legally tolerated in or on food or feed when they are correctly applied [1].

Significant efforts have therefore been directed to the determination of residues in food, with special attention to the development of the so-called green analytical chemistry (GAC) methods [2]. The latter are based on green chemistry principles (e.g. minimizing reagent consumption and waste generation, using safer reagents and miniaturizing analytical systems) [3], and represent a real challenge for analytical chemists. There are a variety of resources to address and reduce environmental pollution caused by the different stages of the overall analytical process. The usual approach focuses on sample collection and preparation, separation, detection, and data evaluation [3].

In the present work, the simultaneous quantification of the fungicides thiabendazole (TBZ), fuberidazole (FBZ), carbendazim (CBZ) and fenarimol (FM), the herbicides dicamba, imazaquin (IMZQ) and norflurazon (NFZ), the insecticides carbaryl (CBL) and methiocarb, and the plant growth activator 1-naphthol (NAP) is attempted in vegetables samples. Table 1 shows structures, functions and dissociation constants of the studied compounds [4-11].

In complex systems such as the ones here investigated, it is usually difficult to develop a selective method without resorting to extensive preparation steps and 
comprehensive sample clean-up. In these cases, it is extremely useful to couple a chromatographic approach, carried out under isocratic conditions, with the chemometric analysis of second-order data. This allows to considerably simplify the sample pretreatment and to significantly reduce the analysis time [12]. Depending on the spectral properties of the analyzed compounds, either diode-array (DAD) [13-15] or fluorescence (FLD) [16,17] detectors are frequently employed. Recently, liquid chromatography (LC) with dual UV and fluorimetric detections was used for the chemometric determination of sex hormones in natural waters and sediments [18]. In the present work, for the first time, fluorescence emission-elution time second-order data are obtained by sequentially exciting the sample at two different excitation wavelengths during the chromatographic run. This procedure was carried out through the adequate control of the FLD software. Thus, in addition to take advantage of the simultaneous trace analysis of agrochemicals from different classes in a single run, we obtained better sensitivity in the fluorimetric detection by irradiating the analytes with optimal excitation wavelengths.

Both the LC-DAD and LC-FLD second-order data matrices were processed by multivariate curve resolution-alternating least-squares (MCR-ALS) [19] algorithm, which allows us to obtain reliable results in the analysis of the studied agrochemicals in spiked vegetable samples.

\section{Experimental}

\subsection{Reagents and solutions}


TBZ, CBL and NAP were purchased from Sigma Aldrich Co. (St. Louis, MO, USA). CBZ, dicamba, IMZQ, NFZ, FM, and methiocarb were obtained from Fluka (Buchs, Switzerland). FBZ was provided by Riedel-de Haën (Seelze, Germany). Acetonitrile $(\mathrm{ACN})$, methanol, phosphoric acid and sodium dihydrogen phosphate monohydrate were obtained from Merck (Darmstadt, Germany). Milli-Q water (Millipore, Bedford, USA) was used in all experiments. Stock solutions of all analytes of about $1000 \mu \mathrm{g} \mathrm{mL} \mathrm{L}^{-1}$ were prepared in methanol. From these solutions, more diluted methanol solutions (around $5 \mu \mathrm{g}$

$\mathrm{mL}^{-1}$ ) were obtained. Working solutions were prepared immediately before their use by taking appropriate aliquots of diluted methanol solutions, drying the solvent under nitrogen and adding mobile phase (see below) to the desired concentrations.

\subsection{Apparatus}

Chromatographic runs were performed on an HP 1200 liquid chromatograph (Agilent Technologies, Waldbronn, Germany) consisting of a quaternary pump, a manual injector fitted with a $20 \mu \mathrm{L}$ loop, a DAD, an FLD, and the HP ChemStation software package for instrument control, data acquisition and data analysis. A C18 Poroshell 120 EC (4.6×100 mm, $2.7 \mu \mathrm{m}$ particle size) column (Agilent Technologies, Santa Clara, CS, USA) was employed.

\subsection{Chromatographic procedure}


The selected mobile phase was a 60:40 (v/v) mixture of ACN and $0.01 \mathrm{~mol} \mathrm{~L}^{-1}$ phosphate buffer ( $\mathrm{pH} 2.8$ ), delivered at a flow rate of $0.6 \mathrm{~mL} \mathrm{~min}^{-1}$ with a chromatographic system operating under isocratic mode.

Data matrices were collected each $1.7 \mathrm{~s}$ using wavelengths from 200 to $500 \mathrm{~nm}$ in steps of $0.5 \mathrm{~nm}$ for the DAD, and each $1.8 \mathrm{~s}$ from 300 to $500 \mathrm{~nm}$ in steps of $1 \mathrm{~nm}$ for the FLD. In this latter case, the excitation wavelength was firstly set at $275 \mathrm{~nm}$ and, after 2.5 minutes of run, it was automatically changed through the software to $228 \mathrm{~nm}$. Both LCDAD and LC-FLD matrices were saved in ASCII format, and transferred to a PC for subsequent manipulation.

\subsection{Calibration and validation samples}

Table S1 of Supplementary data shows the concentrations of analytes used for the preparation of the 15 samples which constituted the calibration set. The concentrations of those analytes which overlap their chromatographic bands (e.g. FBZ, CBZ and TBZ in the FLD chromatogram, and CBL, NFZ and NAP in the DAD one) were provided by a central composite design. The concentrations of the remaining compounds were equally spaced within the working range. The tested concentrations were in the ranges $0-100 \mathrm{ng} \mathrm{mL}^{-1}$ for dicamba, IMZQ, NFZ, methiocarb and FM, 0-50 ng $\mathrm{mL}^{-1}$ for TBZ and CBZ, and 0-10 ng $\mathrm{mL}^{-1}$ for FBZ. These ranges were selected considering the importance of determining low levels of agrochemicals in food samples, and no efforts were made to establish the upper concentration of the linear range.

A validation test set was prepared employing concentrations different than those used for calibration and following a random design. 


\subsection{Real samples}

Mushroom (Agaricus bisporus), crisphead and butter lettuce, alfalfa sprout, cucumber, and celery were purchased from local supermarkets. Representative $5 \mathrm{~g}$ portions of previously chopped and crushed vegetable samples were spiked with the assayed analytes in order to obtain concentrations near or below the corresponding European Union-MRL in each vegetable (Table S2, Supplementary data).

The semi-solid samples were blended and homogenized with $2.5 \mathrm{~mL}$ water in an ultrasonic bath for $10 \mathrm{~min}$. Then, each sample was added with $5 \mathrm{~mL}$ ethyl acetate and centrifuged for $15 \mathrm{~min}$ at $4000 \mathrm{rpm}$. An aliquot of $3 \mathrm{~mL}$ of the organic supernatant was evaporated in a rotary evaporator, the residue was reconstituted with $3 \mathrm{~mL}$ of mobile phase, and filtered by a nylon filter before the chromatographic analysis.

\subsection{MCR-ALS algorithm and software}

A brief description of the MCR-ALS theory is included in the Supplementary data. The data were handled using the MATLAB computer environment [20]. The calculations involved in the mixture resolution by MCR-ALS were carried out using MVC2, a MATLAB graphical interface toolbox which is a new version of that already reported in the literature [21], freely available on the Internet [22].

\section{Results and discussion}




\subsection{General considerations}

Since data processing will be performed through multivariate calibration, the main objective of the classical chromatographic analysis, i.e., baseline resolution of sample components, is not strictly required. In fact, the present work was carried out taking into account the green analytical principles rather than focusing on complete band separation. Thus, isocratic chromatographic conditions were optimized in order to shorten the elution times as much as possible, with the concomitant decrease in consumption of organic solvents.

According to previous experience [23], mobile phases containing different ratios of acetonitrile and buffer solutions were tested. All investigated analytes are organic compounds of relative low polarity, and having different acid-base properties (Table 1). Therefore, the selection of the mobile phase $\mathrm{pH}$ plays an important role in their chromatographic retention times. It was corroborated that mobile phases of $\mathrm{pH}>4$ rendered long run times and, on the other hand, $\mathrm{pH}$ lower than 2 was avoided to extend the C18 column life. Therefore, $\mathrm{pH}$ values between 2 and 4 were tried. A mobile phase constituted by acetonitrile and phosphate buffer pH $2.8(60: 40 \mathrm{v} / \mathrm{v})$, applied in an isocratic

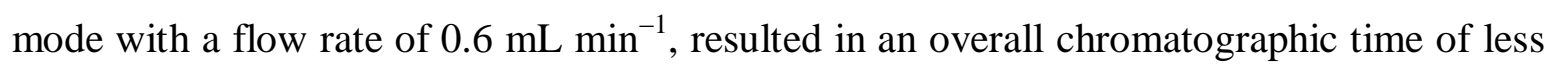
than 5 min per run. Under these conditions, protonated benzimidazolic fungicides (TBZ, FBZ, CBZ) emerge first, followed by dicamba (which, in part, is negatively charged) and the neutral structures of IMZQ, CBL, NFZ, NAP, methiocarb and FM. 
Both LC-DAD and LC-FLD matrices were measured for calibration and validation samples. Fig. 1 shows the DAD and FLD three-dimensional plots for a typical synthetic sample containing the studied analytes.

The obtained second-order data were chemometrically processed with the purpose of determining either partial or total coeluting compounds and, in addition, obtaining the second-order advantage [24]. This latter property allows us to quantify analytes even in the presence of compounds which were absent in the calibration step and, therefore, long and tedious sample clean-up treatments are avoided, with a significant decrease in the use of organic solvents.

UV-fluorescence dual detection was selected because of its analytical advantages: 1) UV signals allow us the determination of a wider number of compounds and, 2) fluorescence signals are, in general, more sensitive and selective than those based on absorption. Unfortunately, LC-DAD and LC-FLD data could not be fused and treated as a single unified matrix. This is due to the delay time between the two modes of detection, causing chromatographic bands registered by both detectors for each analyte to have different shapes.

It is known that second-order data arising from chromatographic measurements with DAD or FLD for complex multicomponent samples can be classified as nontrilinear of type 1, e.g. elution time profiles are not constant from run to run [12]. The three-way array built with nontrilinear data of type 1 for a number of samples can be unfolded into an augmented matrix which preserves the bilinearity property, if the unfolding direction is the one corresponding to profile changes (elution time in the present case). MCR-ALS, a popular algorithm which deals with this situation, was selected to process the data [19]. 
It is necessary to point out that if the UV or fluorescence spectra of the analytes were sufficiently different, the chromatograms could be processed in the total time range. However, in multicomponent systems it is very likeky to find spectral similarities among analytes. In this situation, if the full chromatograms were processed, unsuitable results would be obtained because the mathematical pseudorank would be smaller than the chemical rank [12]. As will be seen below, to overcome this situation, MCR-ALS was applied in selected time ranges, ensuring that each partial chromatographic region includes analytes with different spectral profiles.

For clarity, we will separately discuss the characteristics of the LC-DAD and LCFLD matrices obtained in the system under study. However, we must recall that for the same sample both matrices are obtained in a single run.

\subsection{Elution time-DAD matrices}

Fig. 2A shows the overlapped experimental DAD chromatograms for the components of a typical validation sample, and the corresponding absorption spectra. TBZ, FBZ and CBZ co-elute with a strong injection peak, and thus they were not determined with DAD. The first analyte whose band is separated from the injection peak in the DAD chromatogram is dicamba, followed by IMZQ, CBL, NFZ, NAP, methiocarb and FM, with different overlapping degrees. Among these latter compounds, CBL and NAP possess fluorescent properties, and were also evaluated by fluorescence (see below).

The absorption spectrum of dicamba is very similar to that of FM (Fig. 2A) and, therefore, for data processing the full DAD matrix was divided in two matrices: one involving dicamba, IMZQ, CBL, NFZ and NAP, and the other one methiocarb and FM. 
For MCR-ALS analysis, LC-DAD matrices for each validation sample were augmented with the calibration data matrices. Initialization of the algorithm was done by estimating the so-called purest variables in the spectral domain, and decomposition was then performed by imposing the restrictions of non-negativity in both modes and unimodality in the temporal mode. The number of MCR components was estimated using principal component analysis, and justified considering the presence of analytes and background signals. The selected ALS convergence criterion was $0.01 \%$ (relative change in fit for successive iterations), and in validation samples convergence was achieved in less than 20 iterations. The residual fits for the DAD were about $0.05 \mathrm{mAU}$ (milli absorbance units). After convergence of the ALS optimization, analytes were identified and quantified with the aid of the corresponding pseudo-univariate calibration curves.

Fig. 2B shows the profiles retrieved by MCR-ALS in the temporal and spectral modes for a typical validation sample. As can be appreciated, although the system is very complex, both the chromatographic bands and the spectra are clearly distinguished.

The prediction results for the analyzed compounds corresponding to the application of MCR-ALS to a set of sixteen validation samples (Fig. 3A) are in good agreement with the corresponding nominal values. If the elliptical joint confidence regions (EJCRs) [25] are analyzed for the slopes and intercepts of each plot (inset of Fig. 3A), we conclude that ellipses include the theoretically expected values of $(1,0)$, indicating the accuracy of the used methodology.

The statistical results corresponding to validation samples are completed with the parameters shown in Table 2. It is necessary to point out that the indicated limits of detection have been calculated using the rigorous expression recommended by the International Union of Pure and Applied Chemistry (IUPAC) [26]: 
$\mathrm{LOD}=3.3\left(\mathrm{SEN}^{-2} \sigma_{x}^{2}+h_{0} \mathrm{SEN}^{-2} \sigma_{x}^{2}+h_{0} \sigma_{y c a l}{ }^{2}\right)^{1 / 2}$

where SEN is the component sensitivity, $\sigma_{x}^{2}$ is the variance in the instrumental signal, $h_{0}$ is the sample leverage at zero analyte concentration, $\sigma_{y c a l}^{2}$ is the variance in calibration concentrations, and the factor 3.3 is the sum of $t$-coefficients accounting for type I and II errors (false detects and false non-detects, respectively) at 95\% confidence level [27].

\subsection{Elution time-FLD matrices}

TBZ, FBZ, CBZ, CBL and NAP were determined through their fluorescent signals. It should be noticed that the first three analytes are not interfered by the injection bands in the FLD mode. Fig. 4A shows the experimental FLD chromatograms and the emission spectra of these compounds. As can be seen, two clear regions are distinguished in the time axis: one of them around 2 min (including TBZ, FBZ, and CBZ) and the other one between 3 and 4 min (including CBL and NAP).

With the purpose of improving the sensitivity of the applied method, a new strategy was here implemented: emission fluorescence data were obtained irradiating each sample sequentially at two optimal excitation wavelengths. As can be appreciated in Fig. 5, the excitation bands for the first emerging analytes (TBZ, FBZ, and CBZ) appear in the range of about 260-330 nm, while CBL and NAP have excitation maxima between 200-250 nm. This fact gaves us the possibility to choose an adequate excitation wavelength for each chromatographic region. Specifically, the excitation wavelength was firstly set at $280 \mathrm{~nm}$, favorable to $\mathrm{CBZ}$ which presents the weakest fluorescence signals within its group, and 
after 2.5 min the value was automatically changed to $228 \mathrm{~nm}$, which is appropriate to NAP (less fluorescent than CBL).

In Fig. 4A, the strong chromatographic overlapping among TBZ, FBZ and CBZ bands is clear, as well as a marked spectral similarity between TBZ and FBZ. These facts represent a chemometric challenge but, as will be demonstrated, the problem could be overcome using adequate restrictions during MCR-ALS fitting. On the other hand, the overlapping between CBL and NAP was also resolved through the applied chemometric approach.

The MCR-ALS procedure, including selection of components and used restrictions, was similar to that carried out for the LC-DAD matrices. Additionally, in the first evaluated region, which includes $\mathrm{TBZ}, \mathrm{FBZ}$, and $\mathrm{CBZ}$, it was essential to apply the correspondence constraint for the correct differentiation between TBZ and FBZ spectra [28]. The latter constraint forces analyte sub-profiles in the augmented elution time mode to be zero for samples where it is absent.

The residual fits for the LC-FLD analysis were about 0.01 UF (arbitrary units of fluorescence), which is ca. $1 \%$ with respect to the maximum measured intensity. After convergence of the ALS optimization, quantification of each analyte was performed through the corresponding pseudo-univariate calibration curves. Fig. 4B shows the satisfactory chromatographic and spectral profiles retrieved by MCR-ALS for a typical validation sample, and Fig. 3B displays the predictions for the fluorescent agrochemicals in validation samples. The good results are also corroborated by the statistical parameters of Table 2. In this table, the positive influence of the FLD in the sensitivity of the method is evident, as reflected in LODs between 0.2 and $3 \mathrm{ng} \mathrm{mL}^{-1}$. 


\subsection{Real samples}

The usefulness of the proposed method was tested by analyzing in land cultivated vegetables that are susceptible to contamination by agrochemicals used to promote and/or to protect crops. Although vegetables here evaluated were from regions of intensive agriculture, where agrochemicals are profusely used, the analytes were not detected in these samples. Therefore, spiked samples were prepared and a recovery study was carried out.

Although the MRL values for the investigated agrochemicals are in a wide range of concentrations (e.g. 10-1000 ng g ${ }^{-1}$, Table S2 of Supplementary data), the assayed levels

were not larger than $100 \mathrm{ng} \mathrm{g}^{-1}$. On the other hand, the good LOQs of the proposed method avoided the need of pre-concentration steps for the quantification of lower residues.

Data processing for the real samples proceeded as for the validation samples. MCRALS was applied using a similar strategy, but an additional correspondence constraint was applied concerning the potential interferences. Their profiles in the augmented mode were forced to be zero in the calibration samples.

The good obtained results in terms of recoveries (Table 3) and EJCR accuracy test (Fig. 6) suggest that neither natural constituents nor other foreign compounds which may be possibly present in the studied samples produce a significant interference in our analysis.

\section{Conclusions}

Multivariate curve resolution coupled to liquid chromatography with UV/fluorescence detection has demonstrated to be a robust and capable tool to solve a 
complex mixture of agrochemicals in vegetables following a green strategy. In fact, the ten analyzed compounds were successfully determined in less than 5 min without a complete chromatographic separation and without removing potential interferents of real matrices. The dual detection allows us to determine a larger number of compounds, while the use of two excitation wavelengths in the fluorescence detector improved the sensitivity of the fluorescence detection. Taking into account the low experimentally required time, the good sensitivity and the significant simplicity, we can assert that the proposed method favorably compares with those usually employed in this type of determinations.

\section{Acknowledgments}

The authors are grateful to the Universidad Nacional de Rosario (Project BIO 237), Consejo Nacional de Investigaciones Científicas y Técnicas (CONICET, Project PIP 0163), and Agencia Nacional de Promoción Científica y Tecnológica (ANPCyT, Project PICT 2013-0136) for financially supporting this work. M. D. C. thanks ANPCyT for a doctoral fellowship.

\section{References}

http://ec.europa.eu/food/plant/pesticides/eu-pesticides-database/public/?event= homepage\&language=EN (accessed February 2015).

[2] S. Armenta, S. Garrigues, M. de la Guardia, Green analytical chemistry, Trends Anal. Chem. 27 (2008) 497-511.

[3] A. Molina Díaz, J.F. García Reyes, B. Gilbert López, Solid-phase spectroscopy from the point of view of green analytical chemistry, Trends Anal. Chem. 29 (2010) 654-666. 
[4] G.N. Piccirilli, G.M. Escandar, Partial least-squares with residual bilinearization for the spectrofluorimetric determination of pesticides. A solution of the problems of inner-filter effects and matrix interferents, Analyst 131 (2006) 1012-1020.

[5] https://www.ebi.ac.uk/chembldb/compound/inspect/CHEMBL28971 (accessed February 2016).

[6] A. Prieto, R. Rodil, J.B. Quintana, R. Cela, M. Möder, I. Rodríguez, Evaluation of polyethersulfone performance for the microextraction of polar chlorinated herbicides from environmental water samples, Talanta 122 (2014) 264-271.

[7] A. Laganà, G. Fago, A. Marino, V.M. Penaz, Liquid chromatography mass spectrometry tandem for multiresidue determination of selected post-emergence herbicides after soil column extraction, Anal. Chim. Acta 415 (2000) 41-56.

[8] Webpage Pubchem open Access data base: http://pubchem.ncbi.nlm.nih.gov/compound/ norflurazon\#section=Top (accessed February 2016).

[9] J. Knaak, C. Dary, M. Okino, F. Power, X.F. Zhang, C. Thompson, R. Velez, J. Blancato, Rev. Environ. Contam. Toxicol. 193 (2008) 53-197.

[10] D.M Whitacre (ed.), Reviews of environmental contamination and toxicology, Springer, New York (USA) 2008, vol. 193, p. 164.

[11] A.C. Kumar, A.K. Mishra 1-Naphthol as an excited state proton transfer fluorescent probe for sensing bound-water hydration of polyvinyl alcohol, Talanta 71 (2007) 2003-200. [12] A.C. Olivieri, G.M. Escandar, Practical three-way calibration, Elsevier, Waltham, USA, 2014.

[13] M.J. Culzoni, A.V. Schenone, N.E. Llamas, M. Garrido, M.S. Di Nezio, B.S. Fernández Band, H.C. Goicoechea, Fast chromatographic method for the determination of 
dyes in beverages by using high performance liquid chromatography-Diode array detection data and second order algorithms, J. Chromatogr. A 1216 (2009) 7063-7070.

[14] R.L. Pérez, G.M. Escandar, Liquid chromatography with diode array detection and multivariate curve resolution for the selective and sensitive quantification of estrogens in natural waters, Anal. Chim. Acta 835 (2014) 19-28.

[15] V. Boeris, J.A. Arancibia, A.C. Olivieri, Determination of five pesticides in juice, fruit and vegetable samplesby means of liquid chromatography combined with multivariate curve resolution, Anal. Chim. Acta 814 (2014) 23-30.

[16] S.A. Bortolato, J.A. Arancibia, G.M. Escandar, Non-trilinear chromatographic time retention-fluorescence emission data coupled to chemometric algorithms for the simultaneous determination of 10 polycyclic aromatic hydrocarbons in the presence of interferences, Anal. Chem. 81 (2009) 8074-8084.

[17] F. Cañada Cañada, J.A. Arancibia, G.M. Escandar, G.A. Ibañez, A. Espinosa Mansilla, A. Muñoz de la Peña, A.C. Olivieri, Second-order multivariate calibration procedures applied to high-performance liquid chromatography coupled to fast-scanning fluorescence detection for the determination of fluoroquinolones, J. Chromatogr. A 1216 (2009) 48684876.

[18] R.L. Pérez, G.M. Escandar, Multivariate calibration-assisted high-performance liquid chromatography with dual UV and fluorimetric detection for the analysis of natural and synthetic sex hormones in environmental waters and sediments, Environ. Pollut. 209 (2016) $114-122$. 
[19] R. Tauler, M. Maeder, A. de Juan, Multiset data analysis: extended multivariate curve resolution, in: S. Brown, R. Tauler, B. Walczak (Eds.), Comprehensive Chemometrics, 2, Elsevier, Oxford, 2009, pp. 473-550.

[20] MATLAB version 2011b, The Mathworks Inc., Natick, Massachussets, USA.

[21] A.C. Olivieri, H.L. Wu, R.Q. Yu, MVC2: a MATLAB graphical interface toolbox for second-order multivariate calibration, Chemom. Intell. Lab. Syst. 96 (2009) 246-251.

[22] www.iquir-conicet.gov.ar/descargas/mvc2.rar (accessed February 2016).

[23] M. Asensio-Ramos, J. Hernández-Borges, T.M. Borges-Miquel,.M.A. RodríguezDelgado, Ionic liquid-dispersive liquid-liquid microextraction for the simultaneous determination of pesticides and metabolites in soils using high-performance liquid chromatography and fluorescence detection, J. Chromatogr. A 1218 (2011) 4808-4816.

[24] A.C. Olivieri, Analytical advantages of multivariate data processing. One, two, three, infinity? Anal. Chem. 80 (2008) 5713-5720.

[25] A.G. González, M.A. Herrador, A.G. Asuero, Intra-laboratory testing of method accuracy from recovery assays, Talanta 48 (1999) 729-736.

[26] A.C. Olivieri, Analytical figures of merit: from univariate to multiway calibration, Chem. Rev. 114 (2014) 5358-5378.

[27] C. Bauza, G.A. Ibañez, R. Tauler, A.C. Olivieri, Sensitivity equation for quantitative analysis with multivariate curve resolution-alternating least-squares: theoretical and experimental approach, Anal. Chem. 84 (2012) 8697-8706.

[28] R. Tauler, M. Maeder, A. de Juan, A., Multiset data analysis: extended multivariate curve resolution. In S. Brown, R. Tauler, 
B. Walczak (eds.), Comprehensive Chemometrics, Elsevier, Amsterdam, 2009, vol. 2, p. 473. 


\section{Figure captions}

Fig. 1 Three-dimensional profiles obtained with DAD and FLD for a synthetic sample containing the ten studied agrochemicals dissolved in mobile phase at part-per-billion concentrations. For clarity, FLD data were split in two plots.

Fig. 2 (A) Overlapped experimental chromatograms obtained with DAD at $220 \mathrm{~nm}$ for the studied system under the selected working conditions (top), and the normalized absorbance spectra in the first (medium) and second (bottom) evaluated time regions. (B) Profiles retrieved by MCR-ALS for the same system.

Fig. 3 Predicted concentrations in validation samples as a function of the nominal values for dicamba (brown), IMZQ (violet), CBL (blue), NFZ (pink), NAP (dark green), methiocarb (orange), and FM (gray) using DAD (A), and for TBZ (black), FBZ (red), CBZ (light green), CBL (blue), and NAP (dark green) using FLD (B). The solid lines are the perfect fit. Each inset shows the corresponding elliptic joint confidence regions (at $95 \%$ confidence level) for predictions.

Fig. 4 (A) Overlapped experimental chromatograms obtained with FLD at $\lambda \mathrm{em}=330 \mathrm{~nm}$ for the studied system under the selected working conditions (top), and the normalized fluorescence spectra in the first (medium) and second (bottom) evaluated time regions. The arrow in the chromatogram marks the excitation wavelength change (see text).

(B) Profiles retrieved by MCR-ALS for the same system.

Fig. 5 Normalized excitation spectra for TBZ, FBZ, CBZ, CBL and NAP. Dashed lines indicate the used excitation wavelengths.

Fig. 6 Plot for predicted concentrations of the ten studied agrochemicals as a function of the nominal values in fungus (circle), crisphead (square) and butter (diamond) lettuces, alfalfa sprouts (triangle up), cucumber (triangle down), and celery (hexagon) samples (error bars correspond to duplicates). The inset shows the corresponding elliptical joint region at $95 \%$ confidence level. The cross marks the theoretical (intercept $=0$, slope $=1$ ) point. 


\section{DAD}

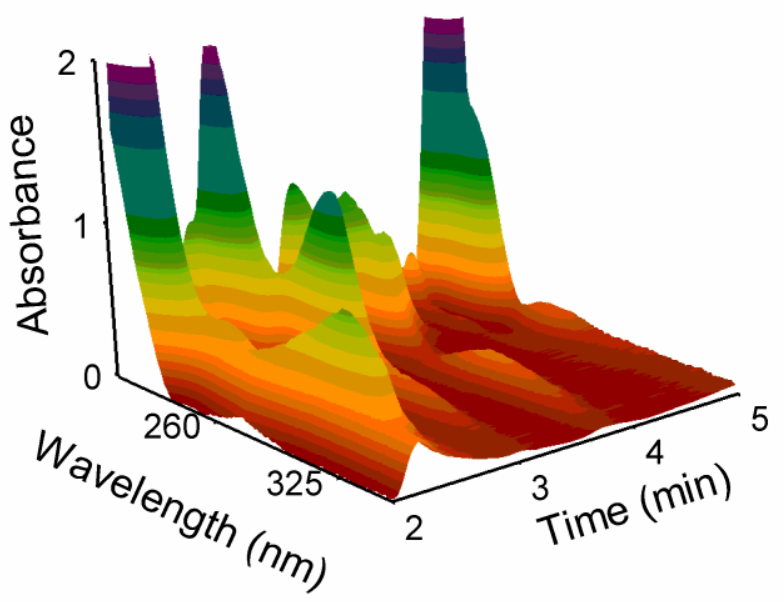

\section{FLD}
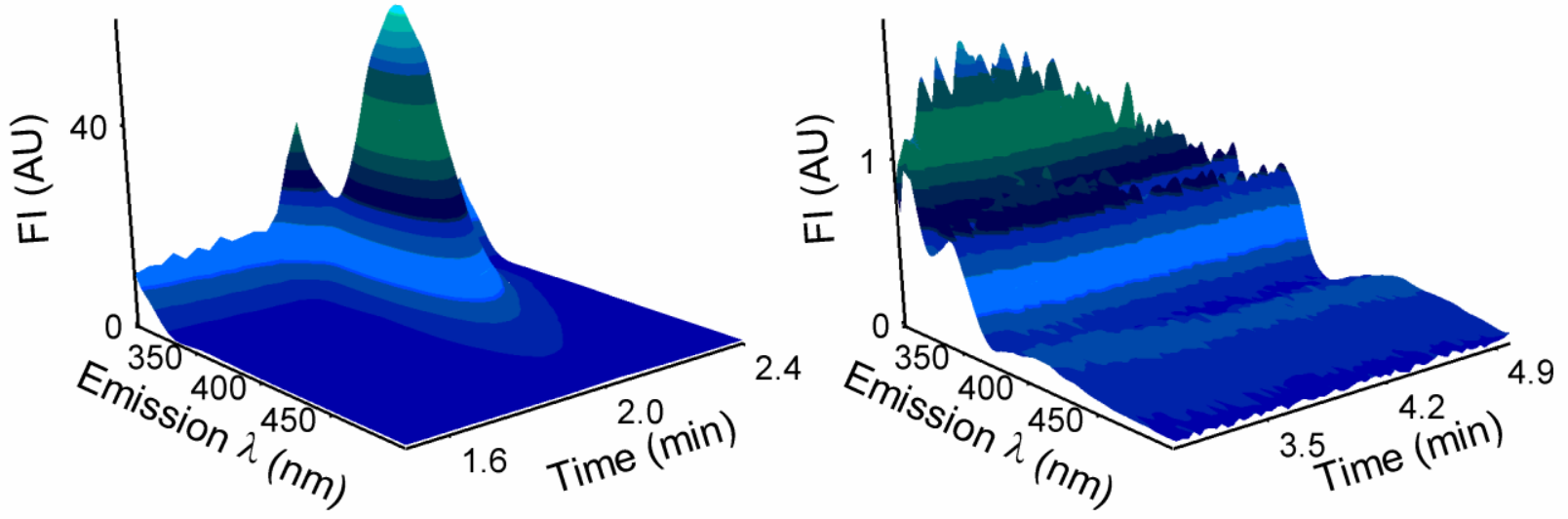

Figure 1 


\section{(A) EXPERIMENTAL}
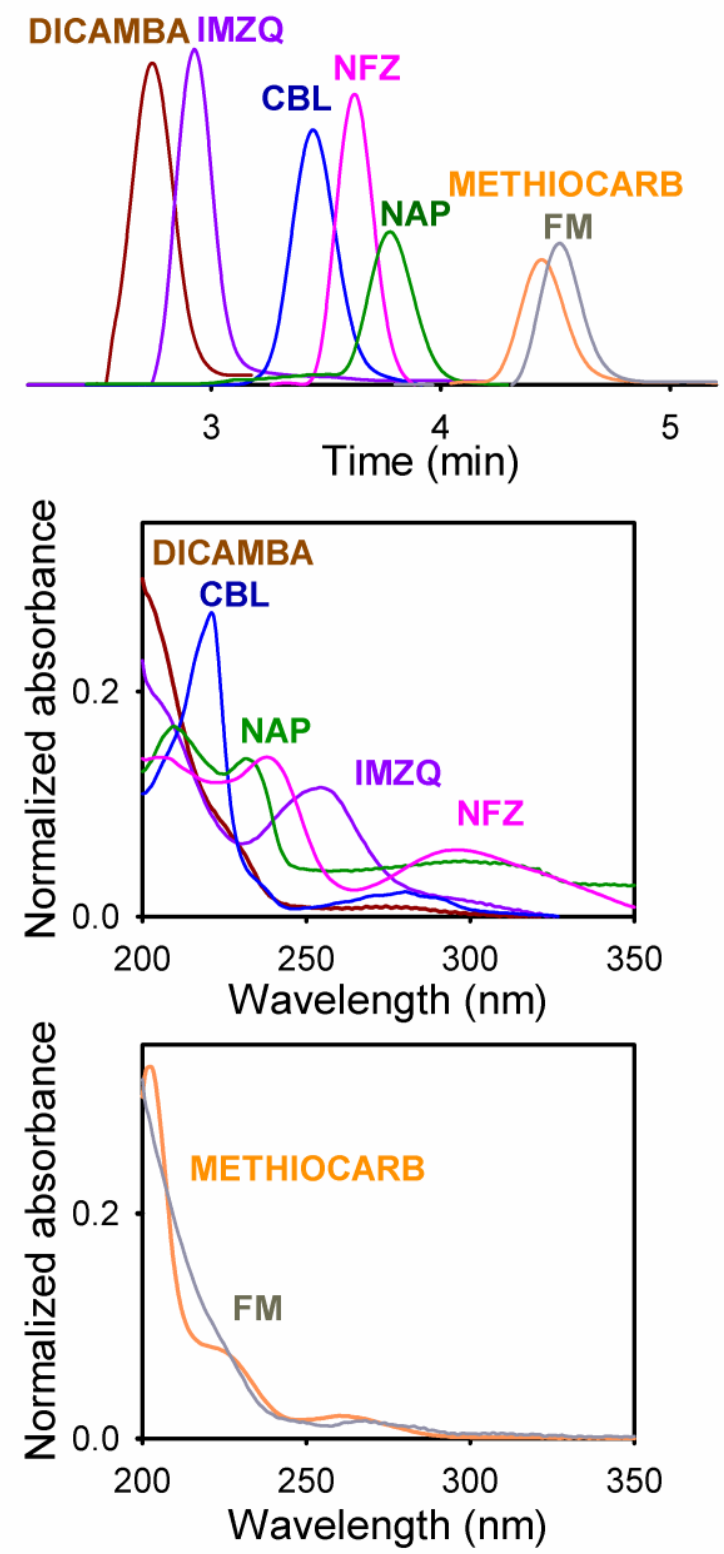

(B) MCR-ALS
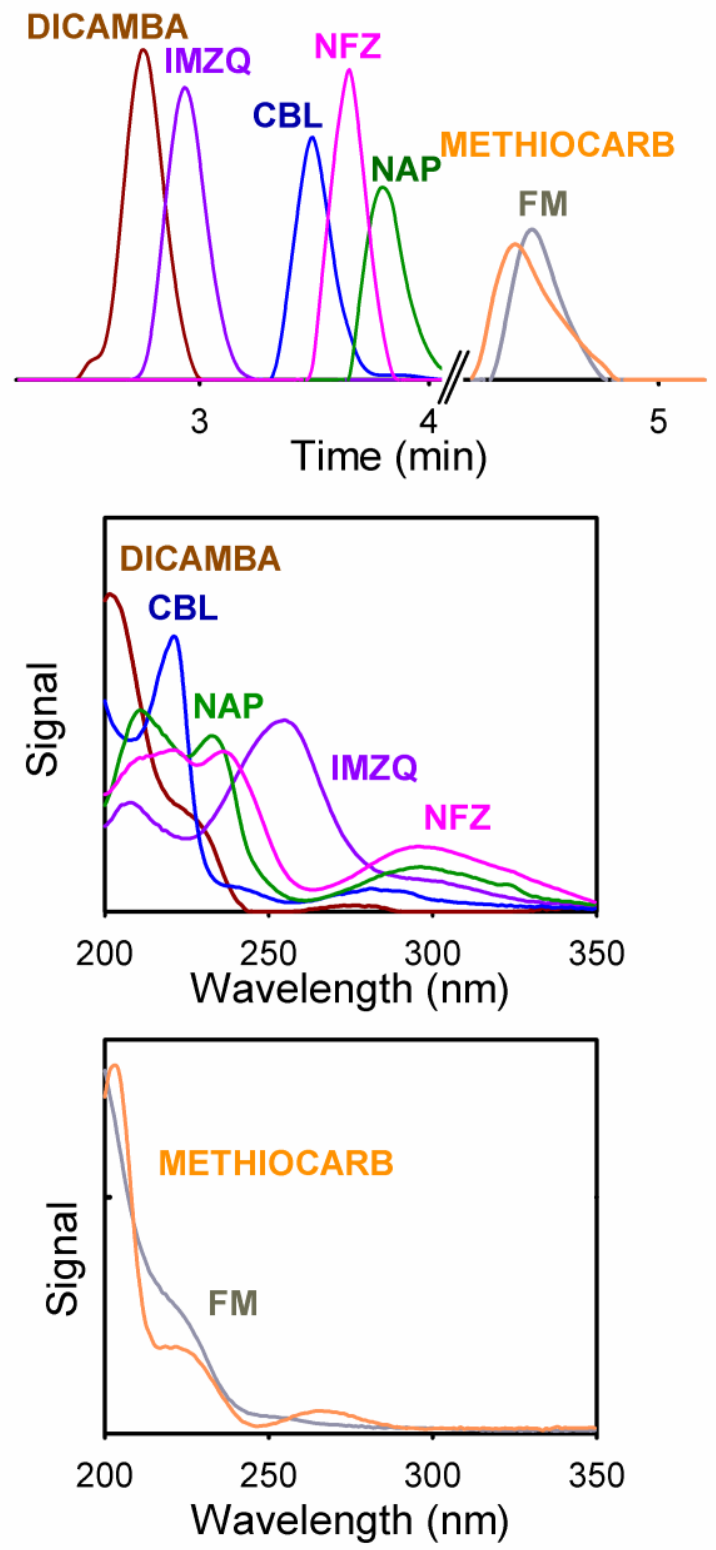

Figure 2 

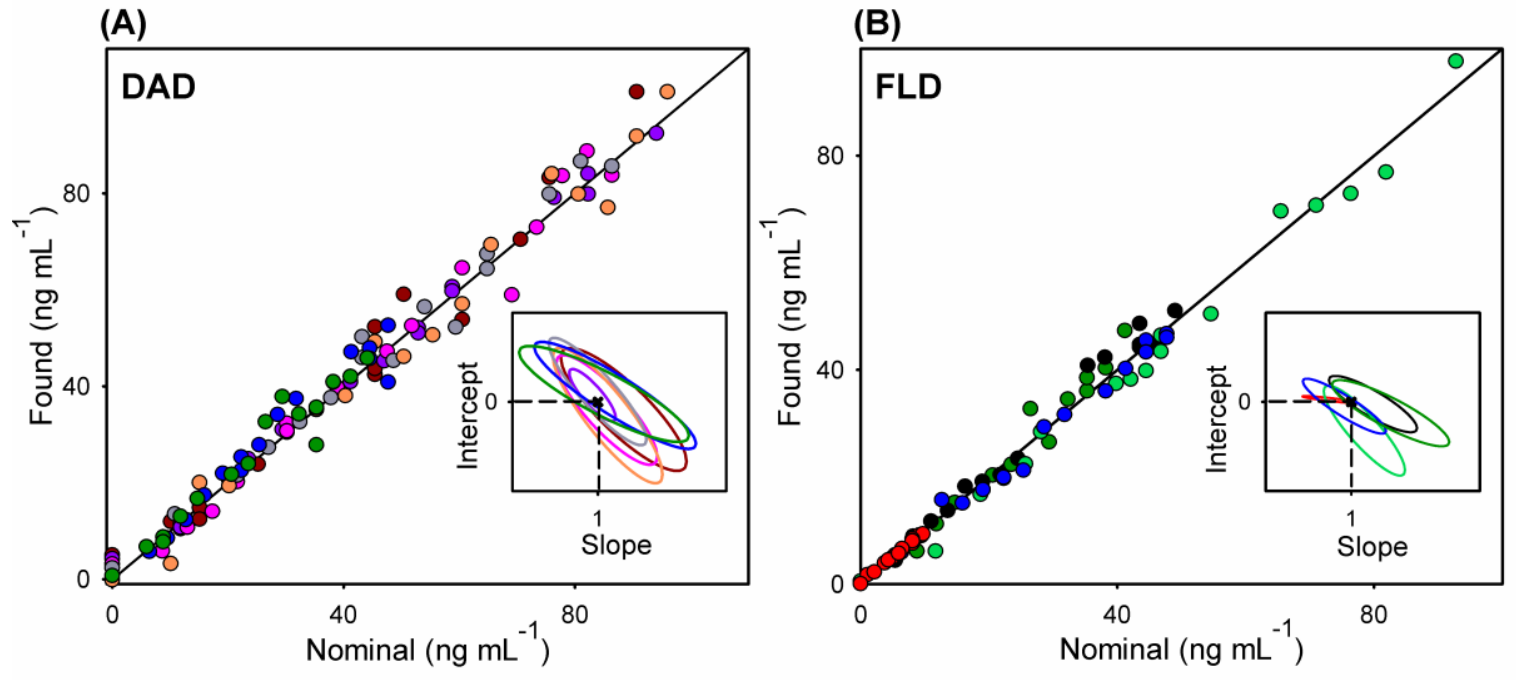

Figure 3 
(A) EXPERIMENTAL
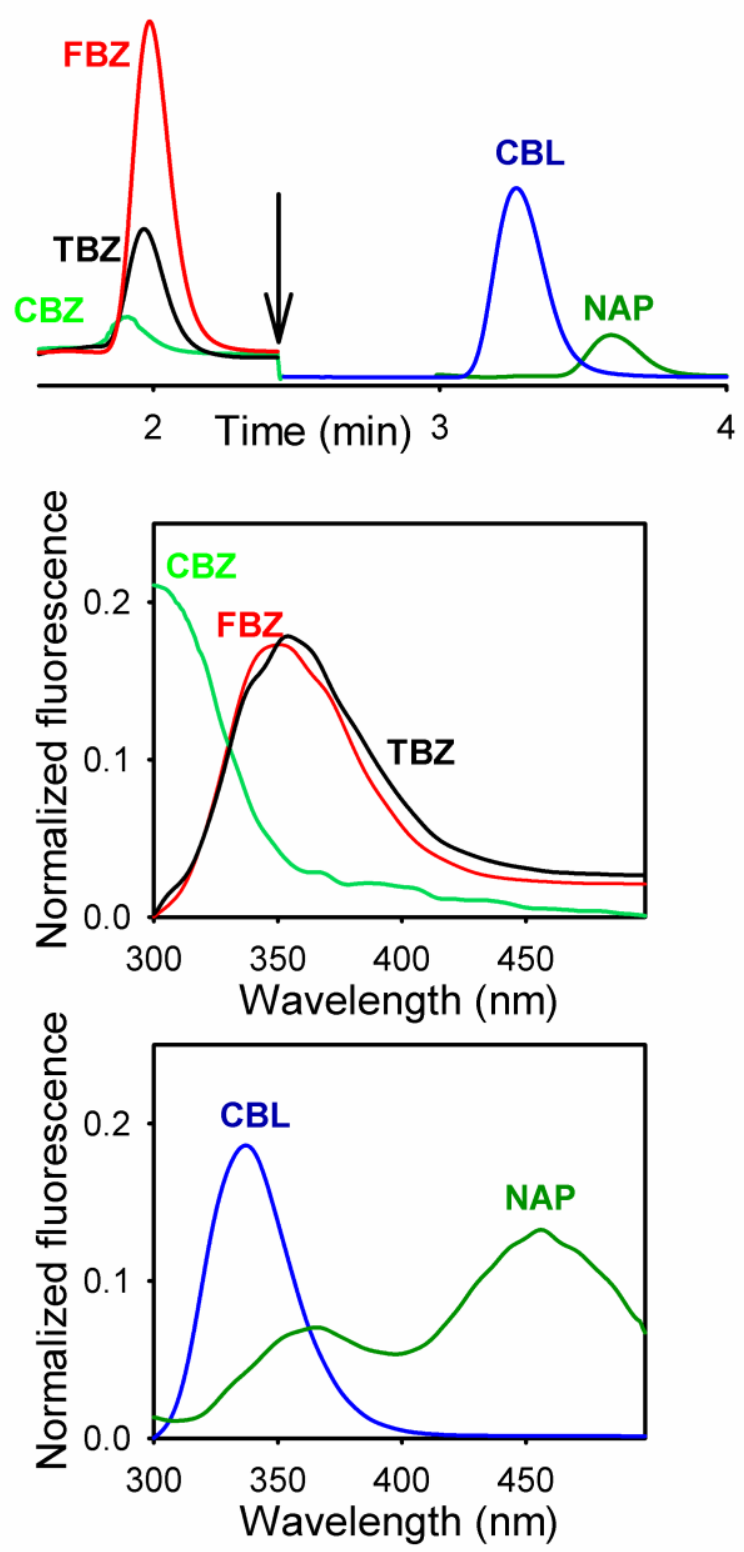

(B) MCR-ALS
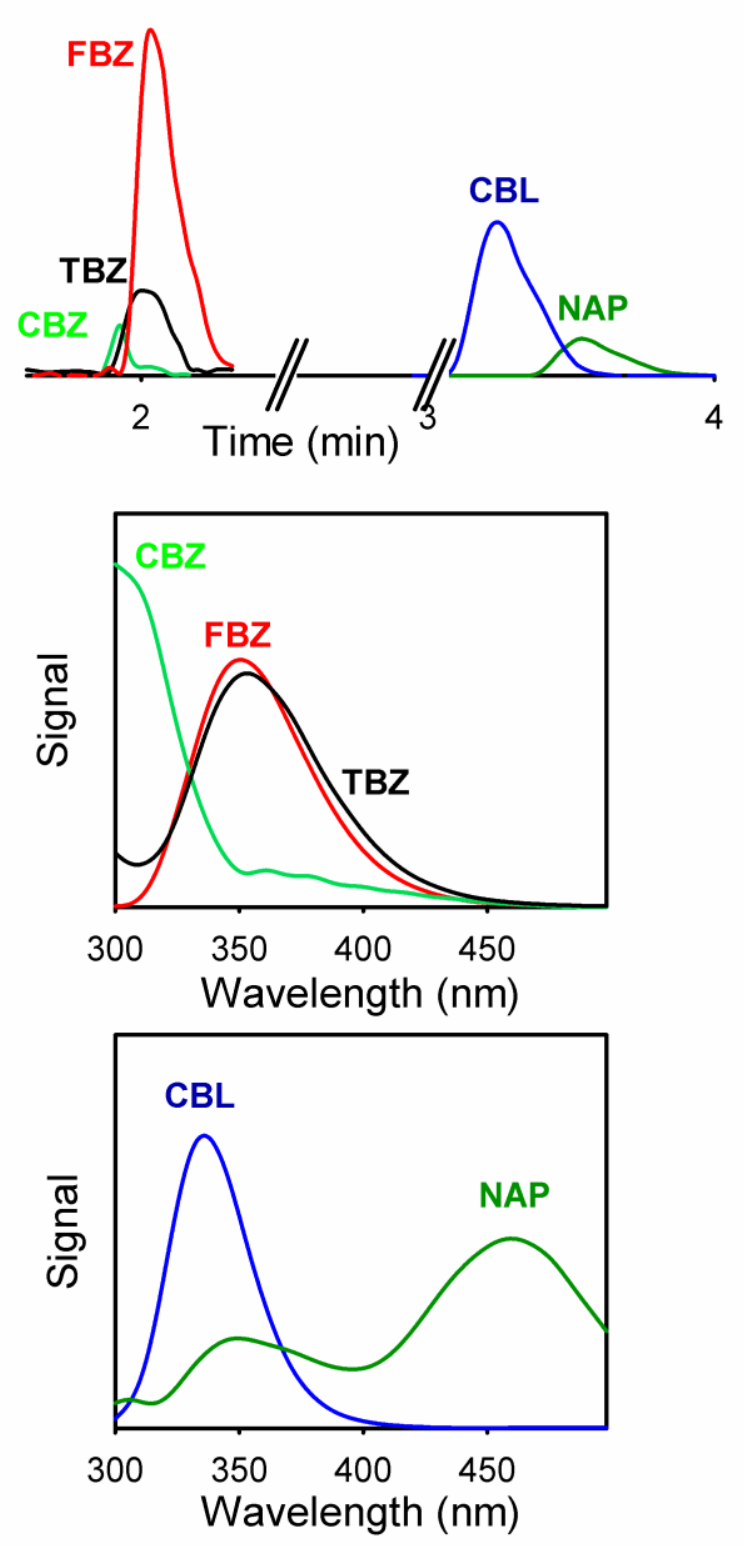

Figure 4 


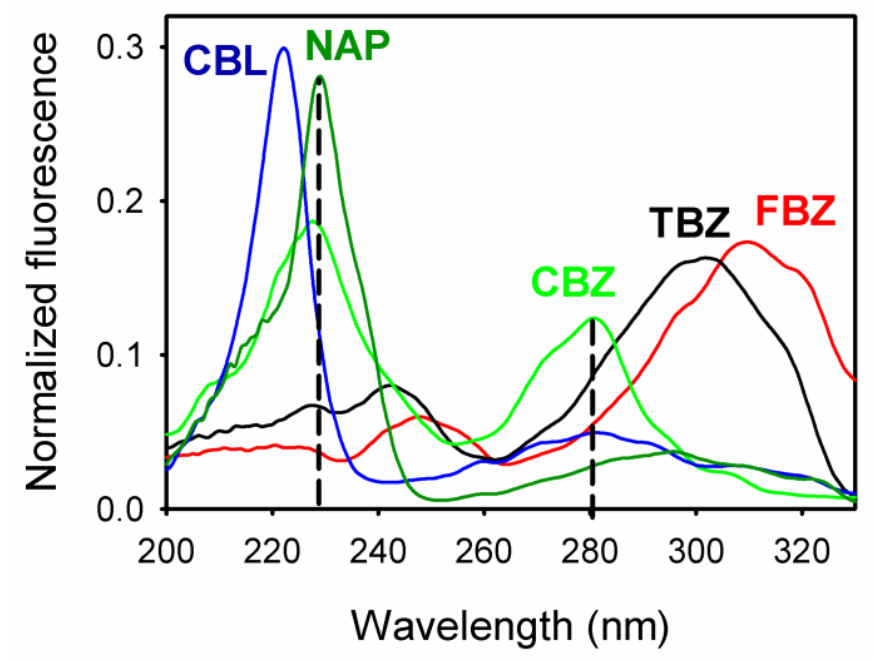

Figure 5 


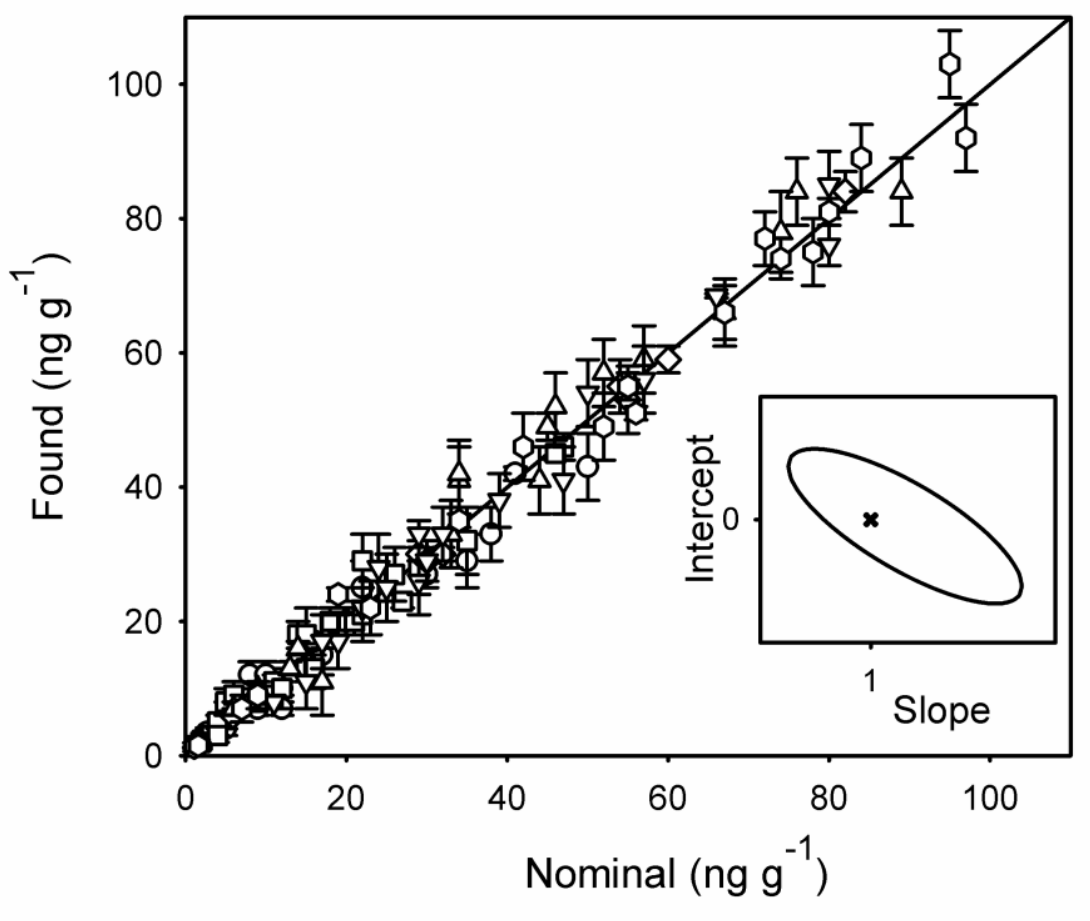

Figure 6 
Table 1

Chemical structures, activities and dissociation constants for the studied agrochemicals

\begin{tabular}{|c|c|c|c|c|}
\hline Name & Activity & Structure & Dissociation constant & Ref \\
\hline TBZ & Fungicide & & $\begin{array}{l}\mathrm{p} K \mathrm{a} 1=0.5 \\
\mathrm{p} K \mathrm{a} 2=4.8 \\
\mathrm{p} K \mathrm{a} 3=11.3\end{array}$ & [4] \\
\hline FBZ & Fungicide & & $\begin{array}{l}\mathrm{p} K \mathrm{a} 1=5.0 \\
\mathrm{p} K \mathrm{a} 2=11.7\end{array}$ & [4] \\
\hline $\mathrm{CBZ}$ & Fungicide & & $\begin{array}{l}\mathrm{pKa} 1=4.3 \\
\mathrm{p} K \mathrm{a} 2=10.8\end{array}$ & [4] \\
\hline FM & Fungicide & & $\begin{array}{l}\mathrm{p} K \mathrm{a} 1=1.67 \\
\mathrm{p} K \mathrm{a} 2=11.23\end{array}$ & {$[5]$} \\
\hline Dicamba & Herbicide & & $\mathrm{p} K \mathrm{a}=2.4$ & {$[6]$} \\
\hline IMZQ & Herbicide & & $\mathrm{p} K \mathrm{a}=3.8$ & [7] \\
\hline NFZ & Herbicide & & $\begin{array}{l}\text { No dissociation in } \mathrm{pH} \\
\text { range } 1-12\end{array}$ & {$[8]$} \\
\hline CBL & Insecticide & & $\mathrm{p} K \mathrm{a}=12.02$ & [9] \\
\hline Methiocarb & Insecticide & & $\mathrm{p} K \mathrm{a}=12.16$ & {$[10]$} \\
\hline NAP & $\begin{array}{l}\text { Plant growth } \\
\text { regulator }\end{array}$ & & $\mathrm{p} K \mathrm{a}=9.4$ & {$[11]$} \\
\hline
\end{tabular}


Table 2

Statistical results for the studied agrochemicals measured with DAD and/or FLD in validation samples ${ }^{\mathrm{a}}$

\begin{tabular}{|c|c|c|c|c|c|c|c|c|c|}
\hline & Dicamba & IMZQ & NFZ & Methiocarb FM & TBZ & FBZ & $\mathrm{CBZ}$ & CBL & NAP \\
\hline \multicolumn{10}{|l|}{ DAD } \\
\hline RMSEP & 5 & 2 & 4 & 5 & & & & 4 & 4 \\
\hline REP & 10 & 4 & 8 & 7 & & & & 14 & 7 \\
\hline LOD & 4 & 2 & 2 & 1 & & & & 2 & 3 \\
\hline LOQ & 12 & 6 & 6 & 3 & & & & 10 & 9 \\
\hline \multicolumn{10}{|l|}{ FLD } \\
\hline RMSEP & & & & & 2 & 0.2 & 4 & 2 & 3 \\
\hline REP & & & & & 9 & 5 & 7 & 7 & 11 \\
\hline LOD & & & & & 1 & 0.2 & 3 & 1 & 1 \\
\hline LOQ & & & & & 3 & 0.6 & 9 & 3 & 3 \\
\hline
\end{tabular}


Table 3

Recovery study for the analytes in spiked real samples using either DAD or FLD and MCR-ALS.

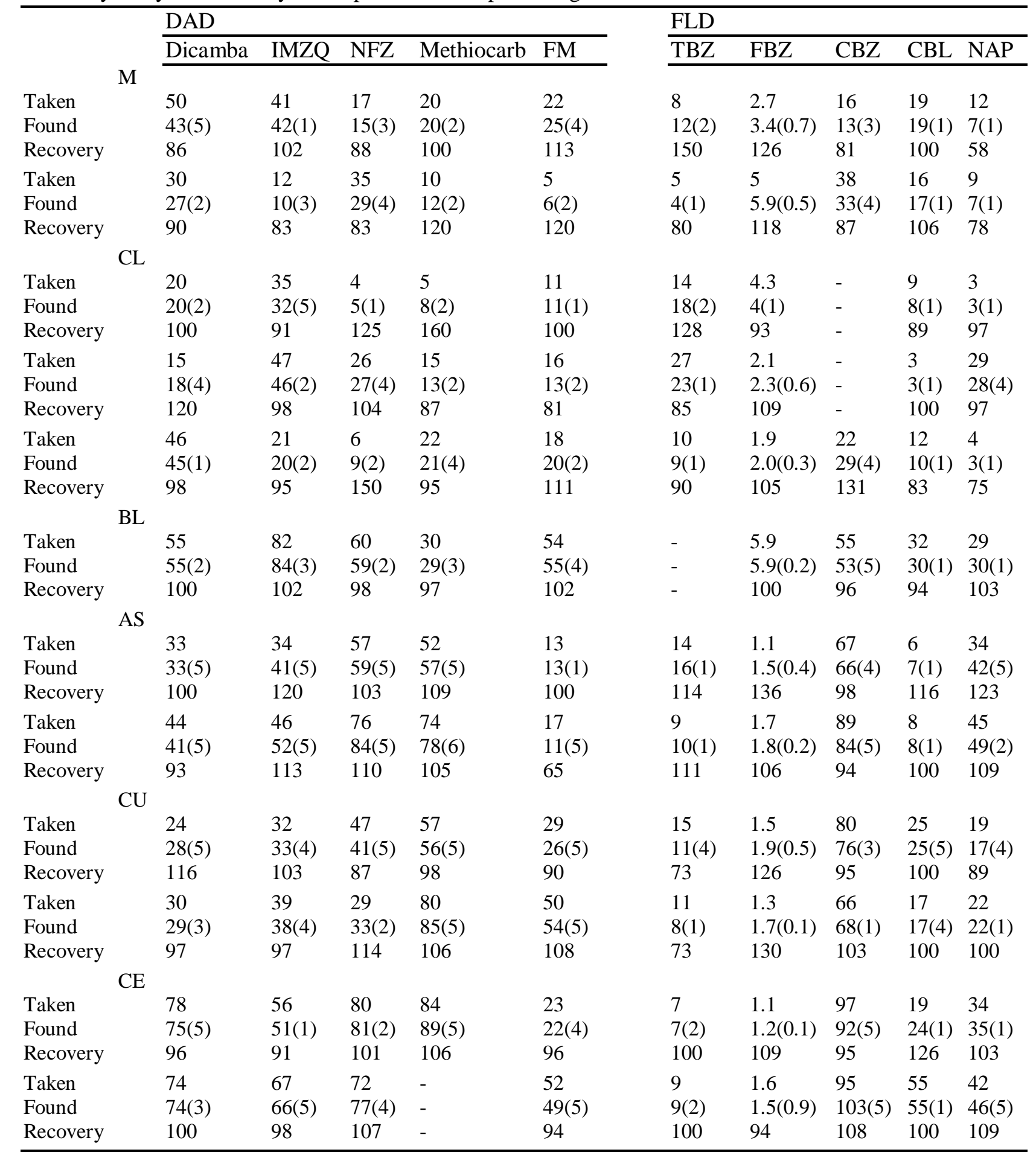

Mushrooms (M), crisphead (CL) and butter (BL) lettuces, alfalfa sprouts (AS), cucumber (CU), and celery (CE) samples. Concentrations are given in $\mathrm{ng} \mathrm{g}^{-1}$ and recoveries are given in percentage. The found values are means of duplicates. Standard deviation between parentheses. 